\title{
Estimating the Sample Size for Training Intrusion Detection Systems
}

\author{
Yasmen Wahba \\ Faculty of Computers and Informatics, Suez Canal University, Ismailia, Egypt \\ E-mail: yasmen.wahba@ci.suez.edu.eg \\ Ehab ElSalamouny and Ghada ElTaweel \\ Faculty of Computers and Informatics, Suez Canal University, Ismailia, Egypt \\ E-mail: \{es, ghada_eltawel\}@ci.suez.edu.eg
}

Received: 14 May 2017; Accepted: 12 September 2017; Published: 08 December 2017

\begin{abstract}
Intrusion detection systems (IDS) are gaining attention as network technologies are vastly growing. Most of the research in this field focuses on improving the performance of these systems through various feature selection techniques along with using ensembles of classifiers. An orthogonal problem is to estimate the proper sample sizes to train those classifiers. While this problem has been considered in other disciplines, mainly medical and biological, to study the relation between the sample size and the classifiers accuracy, it has not received a similar attention in the context of intrusion detection as far as we know.

In this paper we focus on systems based on Naïve Bayes classifiers and investigate the effect of the training sample size on the classification performance for the imbalanced NSL-KDD intrusion dataset. In order to estimate the appropriate sample size required to achieve a required classification performance, we constructed the learning curve of the classifier for individual classes in the dataset. For this construction we performed nonlinear least squares curve fitting using two different power law models. Results showed that while the shifted power law outperforms the power law model in terms of fitting performance, it exhibited a poor prediction performance. The power law, on the other hand, showed a significantly better prediction performance for larger sample sizes.
\end{abstract}

Index Terms-Intrusion detection, Nonlinear regression, Naive Bayes, Learning curve, Power law.

\section{INTRODUCTION}

In machine learning, the process of collecting training samples can be very expensive and scarce especially in clinical studies [1]. Therefore, the need to predict the size of a training sample to achieve a target classification performance has become crucial. This caused the need to study how the learning algorithm or a classifier behaves with respect to various sample sizes. One of the most common approaches to study the behavior of machine learning algorithms for a given dataset is to model the classification performance as a function of the training sample sizes. This function is called the learning curve of the algorithm [2]. This curve can be used to model the classifiers accuracy of identifying the correct class of an input record, or the error rate of mapping a record to an incorrect class, both describing the performance of the classifier in identifying the correct class of a given input. It is important to remark that the performance of a classifier depends on the real class of a given record. In particular, records that belong to majority classes, i.e. classes that include relatively large number of instances in the dataset, may be easily classified while records that belong to minority classes are hardly classified correctly since few examples of these latter classes are introduced in the training set. Therefore in our study we consider multiple learning curves for the classifier, one for each class in addition to an overall curve that describes the average performance of the classifier.

The behavior of an IDS depends on the dataset as well as the type of the underlying classifier. However all IDSs share the property that the classification performance is improved as more training examples are introduced, i.e. as larger training samples are used. Of course this improvement is reflected by the learning curve which therefore can be used for forecasting the performance at larger sample sizes. From a different perspective, the resulting learning curve can be used to determine the size of the training sample required to reach a predefined accuracy [3].

The construction of the learning curve is based on finding a mathematical model/formula that best fits the set of points, which describe the classifier performance at various sizes for the training sample. This process is known as curve fitting [4]. Once a good model is found, it can be used to predict the performance at larger sample sizes by extrapolation [5].

In this paper, we adopted an experimental approach to investigate the relation between the classifier performance and the size of the training sample. More precisely we focus on the simple probabilistic Naïve Bayes classifier and construct its learning curves. For this purpose we use NLS-KDD intrusion dataset and vary the 
training size from $0 \%$ to $50 \%$ of the total size of the original dataset while the rest of the dataset is used for testing. We use two power law models, namely the simple 2-parameter power law and the 3-parameters shifted power law to construct the learning curves of the classifier. We construct a learning curve for every class in the dataset and describe the quality of the resulting curves in terms of two measures: the fitting performance and the prediction performance. The former measure describes the goodness of the fit while the latter describes the quality of the curve in predicting the classifier accuracy at larger sample sizes.

We conduct our experiments and evaluation using JAVA programming language along with the library classes provided by WEKA machine learning tool.

The rest of the paper is organized as follows: Section II presents related work on sample size determination techniques. Section III presents some preliminaries on Naïve Bayes classifier, learning curves, and techniques of curve fitting. Section IV describes the used intrusion dataset. Section V details the process of evaluating the classifier performance, while Section VI describes the construction of the learning curves. In Sections VII and VIII we evaluate the quality of these curves with respect to fitting performance and predictive performance respectively. Finally in Section IX, we conclude our results and describe possible directions for future work.

\section{RELATED WORK}

Improving the performance of intrusion detection systems is a widely studied topic by researchers. Several methods for improving these systems have been introduced using various approaches [6], [7], [8].

One common approach is using more than one classifier for the training phase, i.e., an ensemble of classifiers. Using ensembles of classifiers proved to be an efficient way for improving IDS. For example, the authors of [9] used a new hybrid radial basis (RB) and support vector machines (SVM), while the authors of [10] used SVM with genetic algorithms (GA).

Another research approach focuses on the preprocessing phase prior to classification. This phase involve reducing the number of features either by feature reduction [11], [12], [13], [14], [15], where the number of features is reduced by selecting a set of features from the original feature set, or through feature extraction [16], [17], which produces a new set of features by transforming the original feature space to a smaller feature space, so as to reduce the dimension.

Another way for increasing the efficiency of classification involves using the method of discretization [18], which converts numerical features into nominal ones. This process greatly improves the overall classification performance, in addition to saving storage space since the discretized data requires less space.

We remark that while the above approaches aim at optimizing the performance of the classifiers given a fixed training set, an orthogonal direction to improve the classification performance is to use larger samples for training. The larger training sample is used, the better classification performance is achieved. This raises many research questions about the relation between the training sample size and the performance. Several researches proved the presence of a positive relationship between the training sample size and the classification accuracy [19], [20]. Going in depth into this problem, different methods are used by researchers for the process of sample size determination (SSD). Authors of [21] used the SSD in order to achieve a sufficient statistical power. The statistical power of a test is defined by Cohen [22] as the probability that the test will lead to the rejection of the null hypothesis which is defined as the hypothesis that the phenomenon in question is, in fact, absent.

Other approaches predict the sample size needed to reach a specific classifier performance [23], [24], [25]. Researchers in [26] proposed an algorithm that calculates the minimum sample size required for the evaluation of fingerprint based systems. For the biomedical field, sample size calculation is highly needed. Authors in [27] tried to calculate the necessary sample sizes to test a good classifier and demonstrate how one classifier outperforms another.

In this paper, we address the problem of selecting the sample size to train an intrusion detection system (IDS). Our approach is based on constructing the learning curve of the classifier using two power law models. We compare between the qualities of the two models in terms of fitting and predicting performances.

\section{PRELIMINARIES}

\section{A. Nä̈ve Bayes Classifiers}

A Naive Bayes classifier is a simple classifier that has a probabilistic basis given by the Bayes theorem as follows. For a given input record having a set of features $A=\left\{a_{1}, \ldots, a_{m}\right\}$, let $P\left(C_{i} \mid A\right)$ be the probability that this record belongs to Class $C_{i}$ given that it has the features $A$. Then the Bayes theorem allows evaluating this probability using other probabilities that are easily evaluated incrementally throughout the learning process as follows [28].

$$
P\left(C_{i} \mid A\right)=P\left(A \mid C_{i}\right) P\left(C_{i}\right) / P(A) .
$$

In the above equation $P\left(C_{i}\right)$ is the marginal probability that the input record belongs to $C_{i}$, and is easily evaluated in the training process by measuring the proportion of class $C_{i}$ in the training set. $P\left(A \mid C_{i}\right)$ is the joint probability of the features $A$ given that the input record belongs to the class $C_{i}$. To evaluate this probability it is assumed that the elementary features of $A$ are independent [29] making $P\left(A \mid C_{i}\right)=\prod_{k} P\left(a_{k} \mid C_{i}\right)$. Note here that the probabilities $P\left(a_{k} \mid C_{i}\right)$ are again easily evaluated through the training process by measuring the proportion of records having $a_{k}$ in the records belonging to $C_{i}$ in the training sample.

The Naive Bayes classifier operates as follows. Given a record having features $A$, the classifier uses (1) to 
evaluates the posterior probability $P\left(C_{i} \mid A\right)$ for every class $C_{i}$, and then reports the most likely class. Note that $P(A)$ is constant for all classes and therefore it is easily evaluated as the normalization constant that satisfies

$$
\sum_{i} P\left(C_{i} \mid A\right)=1
$$

We finally remark that the above independence assumption is the reason why the classifier is called 'naive'.

\section{B. Classifier Performance and Learning Curve}

The performance of a classifier with respect to a given class $C$ is typically measured by its accuracy to recognize the records of class $C$ correctly. In this paper we quantify this accuracy by the sophisticated F-measure which takes into account the precision and recall of the classifier. The precision of the classifier with respect to $C$ quantifies how accurate the classifier is when it reports $C$. Its recall quantifies the completeness of $C$ reports, i.e. the number of correctly reported $C$ records relative to the number of all $C$ records in the testing set. In terms of the numbers of true positives $T P$, false positives $F P$, and false negatives $F N$, the precision and recall of the classifier are given by the following equations.

$$
\begin{aligned}
& \text { Precision }=\frac{T P}{T P+F P} . \\
& \text { Recall }=\frac{T P}{T P+F N} .
\end{aligned}
$$

The F-measure of the classifier is a combination of the above two aspects of the classifier accuracy, and is defined as

$$
F-\text { measure }=2 \times \frac{\text { Precision } \times \text { Recall }}{\text { Precision }+ \text { Recall }} .
$$

Note that both the precision and recall vary from 0 to 1 , and therefore it is easy to see that the F-measure has also the same range. Larger values of the F-measure indicate better quality of classification. Extremely, the classifier is 'perfect' when its F-measure is 1, in which case both its precision and recall are also 1.

A learning curve models the relationship between the sample size and the classifier accuracy. When this accuracy is measured by the F-measure as described above, the learning curve can be approximated by the power-law [3] which is given by the following equation.

$$
\operatorname{Acc}(x)=a x^{\alpha}
$$

where $x$ is the sample size, $a$ is a nonzero positive number representing the learning rate, and $\alpha$ is also a nonzero positive number representing the growth speed of the curve. The values for these parameters differ according to the dataset and the classifier used.

Finally, for completeness, we remark that the performance of a classifier can be alternatively measured in terms of the expected error rate instead of its accuracy. In this case it was shown by [30], [31], [32] that the learning curve can be approximated by the inverse power-laws $e(x)=a x^{-\alpha}$.

\section{Curve Fitting}

Curve fitting techniques are generally used to construct a curve (or a function) that fits, i.e. approximates, a set of measures. In our application we use curve fitting to construct the learning curve of the classifier using a set of performance measures at various sizes of the training sample. In order to perform the curve fitting, a model that specifies the shape of this curve must be chosen. In the following we describe two types of fitting, namely 'linear regression' and 'non-linear regression'.

\section{Linear Regression}

Linear regression simply finds a line that best predicts the value of a variable $y$ from the value of another variable $x$. If the relationship between $x$ and $y$ can be graphed as a straight line, then linear regression is the best choice for our data analysis [33].

\section{E. Nonlinear Regression}

When the data points $\left(x_{i}, y_{i}\right)$ do not form a line, nonlinear regression is an appropriate choice. A nonlinear regression model is a parameterized function (i.e. a curve) $f_{\beta}$ that assigns to every $x_{i}$ a predicted value $f_{\beta}\left(x_{i}\right)$ where $\beta$ is a vector of parameters controlling the behavior of the function. The values of $f_{\beta}\left(x_{i}\right)$ are required to be as close as possible to the real data observations $y_{i}$. This is why the goodness of the regression model is measured by the Sum of Squared Errors (SSE) given by the following equation.

$$
S S E=\sum_{i}\left(y_{i}-f_{\beta}\left(x_{i}\right)\right)^{2}
$$

Given a parameterized function $f_{\beta}$, the best-fit curve is defined by the setting of parameters $\beta$ that minimizes the above SSE of the data points. This curve is obtained by a procedure known as the least-squares method (c.f. [34]). This procedure finds the required optimal values of the parameters $\beta$ of the non-linear regression function $f_{\beta}$ in an iterative fashion. It starts with a set of initial values for each parameter, and then adjusts these parameters iteratively to improve the fit (i.e. to reduce the SSE). The initial values for the parameters need not be so accurate, we just need estimates for them. This can be done be examining the model carefully and understanding the meaning of every parameter in the function $f_{\beta}$.

\section{INTRUSION DATASET}

Our dataset is the NSL-KDD dataset [35], which is suggested to solve some of the problems in the original KDD CUP 99 dataset [36]. The records of this dataset have 41 features and are classified into 5 main classes. One of these classes includes the records that reflect the 
normal traffic and the rest of the classes correspond to different types of attacks. These attacks fall into the following four main categories.

- Denial of Service (DoS): Attacks of this type aim to suspending services of a network resource making it unavailable to its intended users by overloading the server with too many requests to be handled.

- Probe attacks: In these attacks the hacker scans the network aiming to exploiting a known vulnerability.

- Remote-to-Local (R2L) attacks: Here an attacker tries to gain local access to unauthorized information through sending packets to the victim machine.

- User-to-Root (U2R) attacks: Here an attacker gains root access to the system using his normal user account to exploit vulnerabilities.

In our experiments we extracted 62,984 records from the NSL-KDD dataset, where $53 \%$ of the records are normal, and the remaining $47 \%$ are distributed over the four attack types (DoS, PROBE, R2L, U2R). The distribution of the attack classes in the extracted sample is shown in Figure 1. Through the rest of this paper we will always refer to the extracted sample as the dataset.

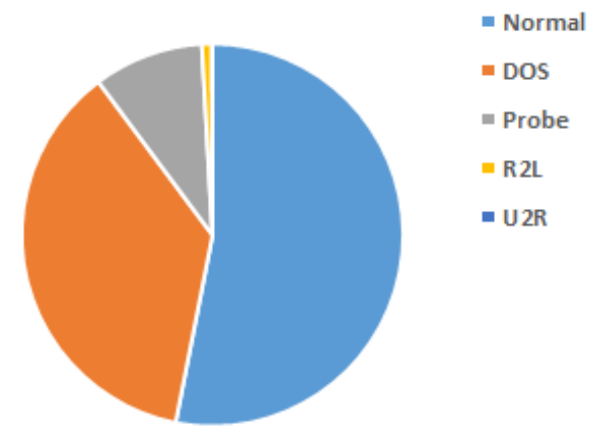

Fig.1. Class Distribution for the NSL-KDD Intrusion Dataset

It is easy to observe from Figure 1 that our NSL-KDD dataset suffers from severe imbalance. The R2L and U2R classes are under-represented, while Normal, DoS and Probe classes represent the majority of the instances. Unbalanced or skewed datasets is one of the major problems that face many researchers when using learning curves to predict the minor classes. In terms of detection accuracy, the detection rate for the minor classes is very poor or sometimes none [11].

This problem is very obvious when plotting the learning curve, where the accuracies for the minor class are very low or even zero at some given sample sizes. The training points are found to be very scattered, thereby making the fitting process hard or sometimes not possible.

\section{Performance of NaÏVE BAyes Classifiers}

In this section we describe the performance of Naïve Bayes classifiers with respect to individual classes in the NSL-KDD dataset. More precisely we describe for each class $C$ in our dataset the accuracy of the classifier to correctly classify a record that belongs to the class $C$. Of course this accuracy depends on the size of training sample, i.e. the number of training examples, and also the popularity of the questioned class in these examples. We use the F-measure (described in Section III-B) to measure the accuracy of the classifier relative to every class.

To investigate the impact of the sample size on the performance of the classifier with respect to each class, we vary the sample size $\varepsilon$ from $1 \%$ to $50 \%$ of the total dataset size and for every value of $\varepsilon$ we perform the following procedure.

1) For $i=1,2, \ldots, 10$ do steps 2 to 4 .

2) Draw a random sample $S_{i}$ of size $\varepsilon$ records from the dataset.

3) Train a Naive Bayes classifier $B_{i}$ on $S_{i}$.

4) Using the remaining records in the dataset for testing, evaluate the accuracy of $B_{i}$ with respect to each class.

5) For each class $C$ evaluate the average accuracy of the 10 classifiers $B_{1}, \ldots, B_{10}$ with respect to the class $C$ and plot this average accuracy against the sample size $\varepsilon$.

The above experiment yields five plots where each one corresponds to one class $C$ and describes the relation between the training sample size and the average accuracy (measured by the f-measure) of correctly detecting records of $C$. Figure 2 displays these plots together with an additional plot which displays the weighted average accuracy for all the classes. This procedure is implemented using the JAVA language along with WEKA library [37] as a data mining tool.

It is important to observe from Figure 2 that the classification performance for minority classes, i.e. R2L and $\mathrm{U} 2 \mathrm{R}$ is very poor compared to other classes. This is because the minority class is relatively unlikely to appear in the training sample and therefore the classifier is unable to learn well to detect such a class. Note also that for all classes the small training samples exhibit bad and unstable performance due to the lack of enough examples that allow the classifier to detect the classes reliably.

\section{CONSTRUCTING THE LEARNING CURVE}

In the previous section, we described our experiments to evaluate the classifier performance given random training samples of variable sizes. In the following we aim to find an approximate relation between the training sample size and the classifier performance. More precisely we use curve fitting techniques to construct the learning curve of the classifiers, which establishes the required relation. 
For the fitting process, we used two versions for the power law model. The first model is the two-parameter power law described in (5) while the second one is the shifted power law having 3 parameters. These two models are summarized in Table 1.

Table 1. Power-law Regression Models

\begin{tabular}{|c|c|}
\hline Model Name & Formula \\
\hline Power Law & $\mathrm{a} \mathrm{x}^{\boldsymbol{\alpha}}$ \\
\hline Shifted Power Law & $\mathrm{a}(\mathrm{x}-\mathrm{b}) \boldsymbol{\alpha}$ \\
\hline
\end{tabular}

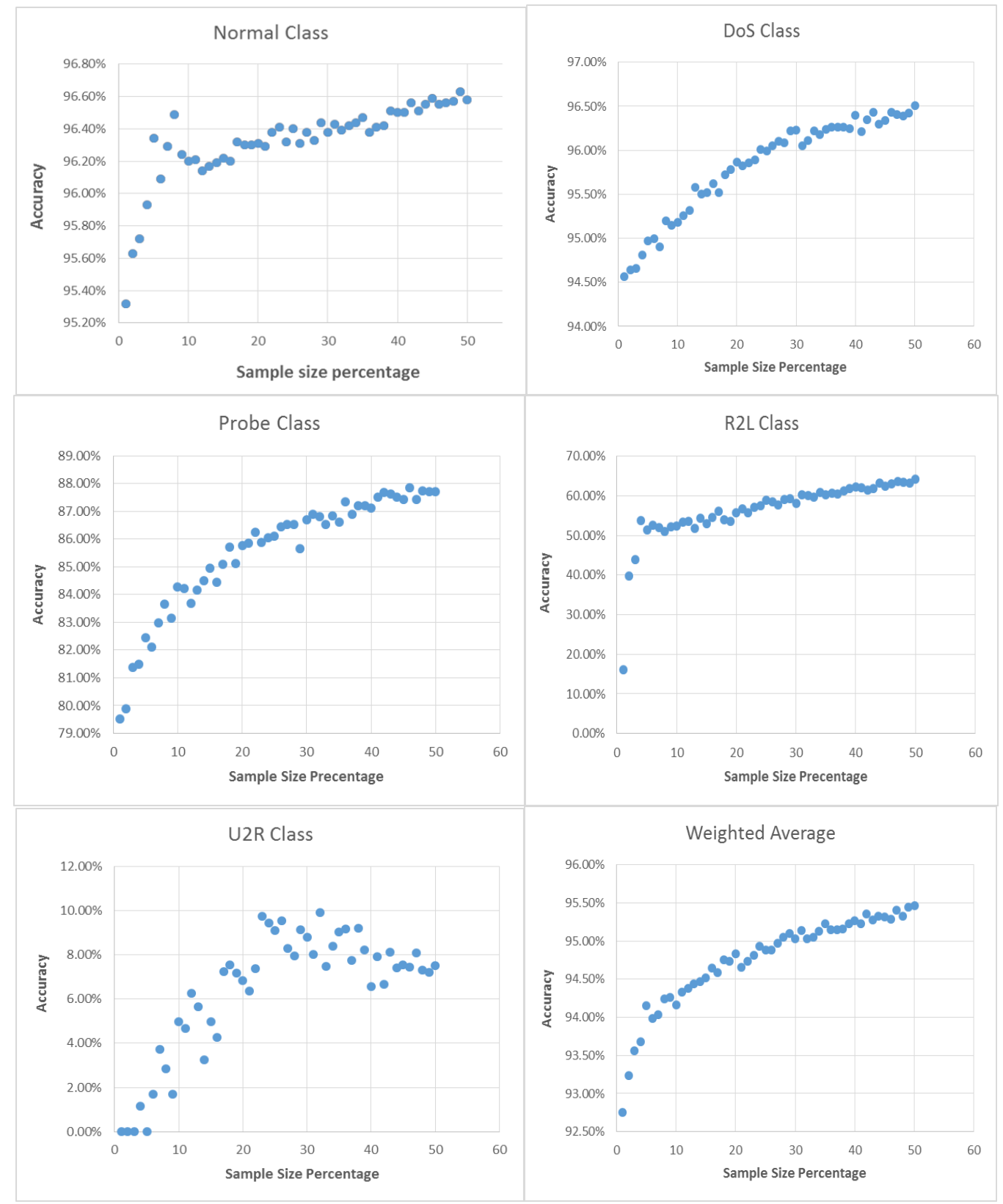

Fig.2. Performance of Naive-Bayes Classifier for Individual NSL-KDD Classes

Based on the performance data that we demonstrated in Figure 2 we construct the learning curve for every class using the two non-linear regression models in Table 1. More precisely, we fitted $50 \%$ of the full-length dataset with respect to every class in our intrusion dataset. Figure 3 demonstrates the results of the curve fitting applied to every class in the dataset.

A first observation from Figure 3 is that the learning curve varies from one class to another. More precisely, the naïve Bayes classifier that learns from a given training sample has variant accuracies to recognize various classes. For example, using $10 \%$ of the dataset for training the classifier, it can recognize the Normal class with accuracy 0.962 , while it recognizes the less common R2L class only with accuracy 0.5. Of course these variations are due to the different proportions of these classes in the training set. Majority classes, e.g. Normal and DoS, are learnt at a higher rate compared to minority classes, e.g. R2L and U2R. However, all curves share a monotonicity property that the accuracy improves when larger training samples are used. 

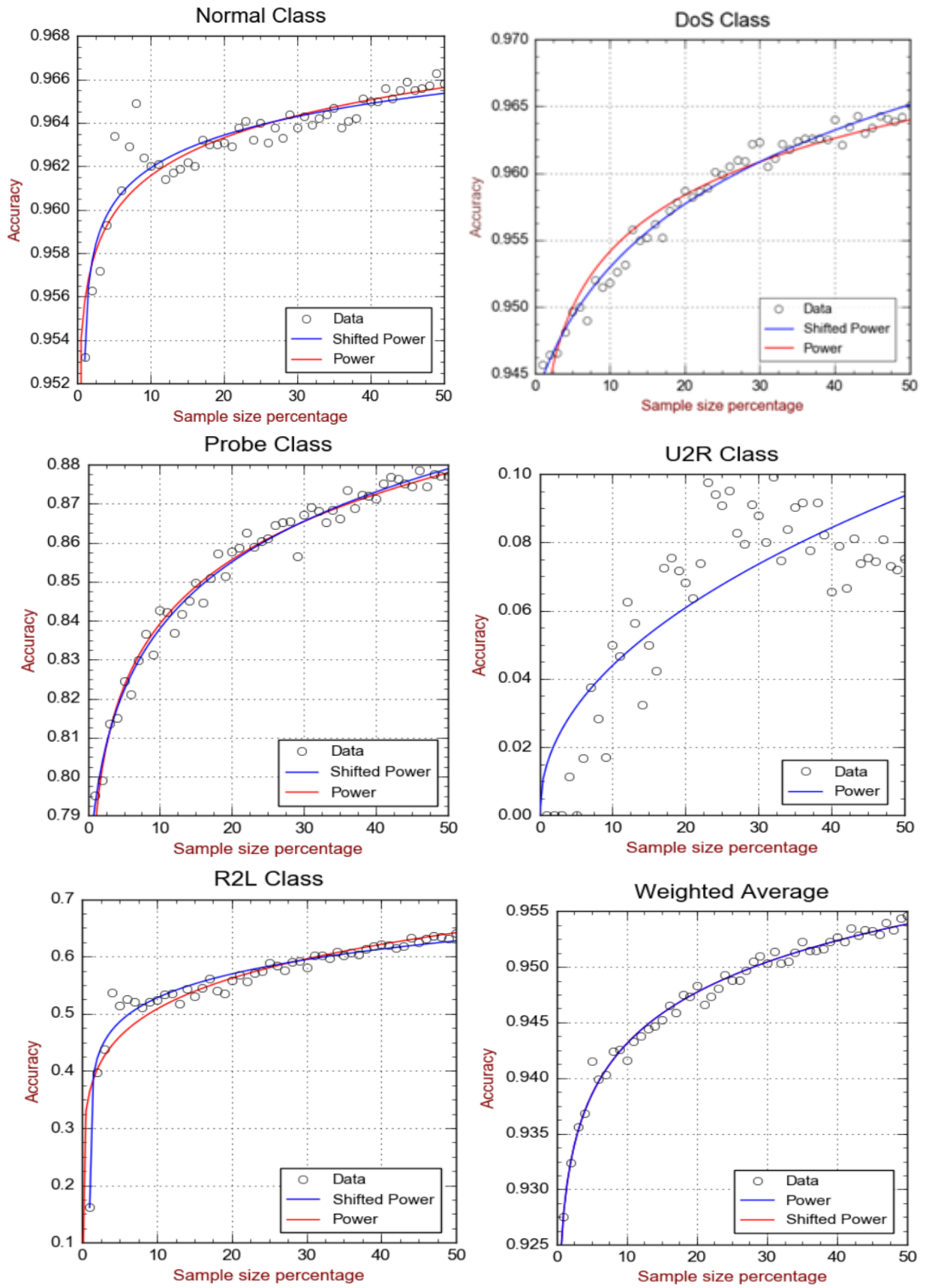

Fig.3. Learning Curves for the Classes of the NSL-KDD Dataset

It can be also seen from Figure 3 that the growth of the learning curve (with the training sample size) varies from one class to another. In particular, the accuracy for the classes Normal grows from 0.962 at sample size $10 \%$ to 0.966 at $50 \%$, i.e. gaining only improvement of 0.004 . However the accuracy for the minority class R2L grows from 0.5 at sample size $10 \%$ to 0.65 at $50 \%$, i.e. gaining a larger improvement of 0.15 . This is again due to the majority of the Normal class compared to R2L. In fact $10 \%$ of the training set is sufficient for the classifier to learn the majority class well such that more introduced examples do not much improve such learning. This situation is clearly different for the minority class R2L, where enlarging the training sample significantly improves the learning quality.
When the target class is a rare or minor, the task of obtaining a good accuracy estimate needs an excessive sample sizes. This is clear in the U2R class, where the number of records is very low in the given training samples. This causes the points of the data points to be very scattered (as seen in Figure 2), and therefore significantly lower the quality of the learning curve. In order to tackle the problem of the low detection rates for both R2L and U2R classes, we applied both sampling methods (Over-sampling and Under-sampling). However, results showed no improvement in the detection accuracy for both those classes.

Researchers in [38] showed that the problem of detecting U2R and R2L cannot be simply solved by resampling techniques since the problem is not directly 
related to the class imbalance. The unsatisfactory detection results were explained using a matrix plot, shown in Figure 4, which showed a clear overlap between these two attack classes. As a consequence of this overlapping, the decision boundaries of these two rare classes could not be generalized clearly by the learning algorithm. This shows that sometimes it is not worth the effort to collect more samples for a given class.

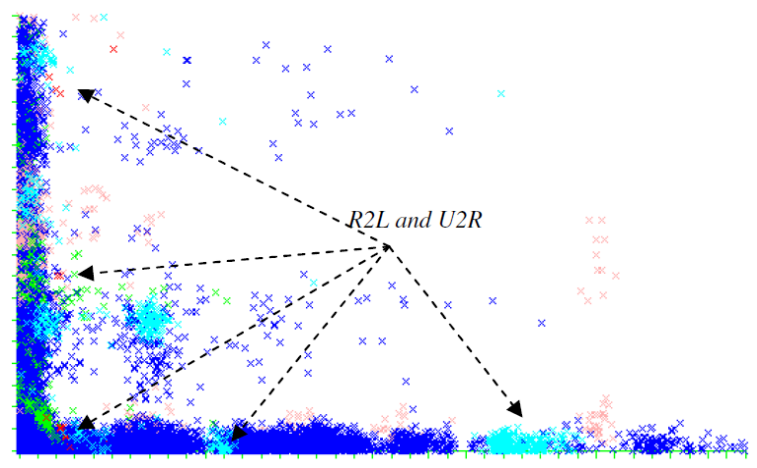

Fig.4. Class overlap problem for U2R and R2L [38]

Finally we remark that the weighted average learning curve is clearly impacted by the major classes and masks the poor quality of the classifier with respect to minority classes. This proves the importance for studying the learning curve for individual classes, instead of studying only the average learning curve.

\section{FITTING PERFormanCE (GOODNESS OF FIT)}

In the following we compare between the goodness of the two non-linear regression models listed in Table 1 . In this comparison we use the following two measures.

- Residuals Standard Error (RSE) [39]: This error is evaluated as

$$
R S E=\sqrt{\frac{S S E}{D F}},
$$

where SSE is the Sum of Squared Errors given by (6) and DF is the degree of freedom, defined as the number of dimensions in which a random vector might vary [40]. For nonlinear regression models, DF can be defined as a measure of optimism of the residual sum of squares (RSS) [41]. It is calculated as the number of data points minus the number of parameters for our model [34].

- R-squared [42]: It is a statistical measure known also as the coefficient of determination. Similar to SSE and RSE, it measures how close the data is to the fitting regression curve. Precisely, it is defined as

$$
R^{2}=1-\frac{S S E}{S S T}
$$

where SSE is the Sum of Squared Errors (6) and SST is the Total Sum of Squares which is the sum of squared deviations of the data measures $y_{i}$ from their mean $\bar{y}$, i.e.

$$
S S T=\sum_{i}\left(y_{i}-\bar{y}\right)^{2}
$$

Note that $\mathrm{R}^{2}$ quantifies the goodness of fitting the data relative to SST which quantifies the variability of the data itself from its mean. Note also that in contrast to RSE, the value of $\mathrm{R}^{2}$ increases when the regression is improved.

For the sake of comparison between the qualities of the two regression models in Table 1, we evaluated the RSE and $\mathrm{R}^{2}$ for all learning curves that we constructed in Section VI for individual classes in the intrusion dataset. The resulting values are shown in Tables 2 and 3.

It is clear from these tables that the shifted (3parameters) power law outperforms the simple power law model in terms of both RSE and R-squared. This is explained by the fact that the shifted power-law has more parameters than the power-law making the former more flexible and hence yielding a curve that is closer to our data points. We remark that in the case of the U2R class the regression based on the shifted power law failed to converge due to the very low number of records of this class in all training samples, making the estimation of the three parameters of the model unstable.

\section{PREDECTION PERFORMANCE}

The technique of predicting the performance of a model at data beyond the available observations is known as extrapolation. Applying this technique to our problem allows us to use the learning curve that is constructed on a range of training sample sizes to predict the quality of the classifier when trained on larger samples. This curve can be also used to estimate the appropriate size of the training sample in order to reach some desired criteria, e.g. obtaining a certain accuracy of detecting a given attack class, or reaching a minimum value for the overall

\begin{tabular}{|c|c|c|c|c|c|c|}
\hline & \multicolumn{6}{|c|}{ Residual Standard Error } \\
\hline & Normal & DoS & Probe & $\mathrm{R} 2 \mathrm{~L}$ & $\mathrm{U} 2 \mathrm{R}$ & $\begin{array}{l}\text { Weighted } \\
\text { Average }\end{array}$ \\
\hline Power Law & 0.001053 & 0.001349 & 0.00320 & 0.03561 & 0.0163 & 0.0007 \\
\hline $\begin{array}{c}\text { Shifted } \\
\text { Power Law }\end{array}$ & 0.000941 & 0.000787 & 0.00299 & 0.01581 & ---- & 0.0007 \\
\hline
\end{tabular}
accuracy.

Table 2. The Fitting Performance of Power-Law Models in Terms of RSE 
Table 3. The Fitting Performance of Power-Law Models In Terms of $\mathrm{R}^{2}$

\begin{tabular}{|c|c|c|c|c|c|c|}
\hline & Normal & DoS & Probe & $\begin{array}{l}\text { R2L } \\
\text { U2R }\end{array}$ & $\begin{array}{c}\text { Weighted } \\
\text { Average }\end{array}$ \\
\hline $\begin{array}{c}\text { Power } \\
\text { Law }\end{array}$ & 0.82 & 0.94 & 0.97 & 0.79 & 0.67 & 0.98 \\
\hline $\begin{array}{c}\text { Shifted } \\
\text { Power } \\
\text { Law }\end{array}$ & 0.86 & 0.98 & 0.98 & 0.95 & ---- & 0.98 \\
\hline
\end{tabular}

It should be noted that the fitting performance for a model might be deceptive. In other words a model might exhibit a high fitting performance but however a very poor predictive performance. This is known as the problem of 'overfitting'. More precisely the model is said to be 'overfitting' when it significantly fits the training data but yields weak estimates when it is tested on new observations [4].

In order to evaluate the prediction performance of a fitting model, we use subsets of only a small portion, e.g. $10 \%$ of the dataset to construct a learning curve, based on the given model, and then examine the ability of the resulting curve to extrapolate larger samples, i.e. its ability to fit the actual accuracy of the classifier when it is trained on larger samples. More precisely we measure the absolute difference between the predicted accuracy at a large sample size using the curve and the real accuracy obtained by training the classifier on a sample of the same large size. The average difference (considering all large sample sizes) is called the prediction error for the model. The model that yields the less prediction error is the better.

In our experiments we restricted the construction of the learning curves to variant small portions of the dataset, specifically $10 \%, 20 \%, 50 \%$, and in each case we evaluated the prediction error of the model for every class in the dataset using the aforementioned procedure. Table 4 demonstrates the results of this evaluation for the power-law model. We also applied the same evaluation method to the more flexible shifted power-law model for which the prediction errors are demonstrated in Table 5.

Table 4. The Prediction Errors of the Power-law Model

\begin{tabular}{|c|c|c|c|c|c|c|}
\hline & \multicolumn{5}{|c}{ Power Law } \\
& Normal & DoS & Probe & R2L & U2R & $\begin{array}{l}\text { Weighted } \\
\text { Average }\end{array}$ \\
\hline $10 \%$ & 0.007 & 0.005 & 0.004 & 1.44 & 0.28 & 0.0006 \\
\hline $20 \%$ & 0.003 & 0.0006 & 0.003 & 0.19 & 0.06 & 0.0005 \\
\hline $50 \%$ & 0.001 & 0.0008 & 0.003 & 0.05 & 0.03 & 0.0005 \\
\hline
\end{tabular}

Table 5. The Prediction Error of Shifted Power-law Model

\begin{tabular}{|c|c|c|c|c|c|c|}
\hline & \multicolumn{5}{|c|}{ Shifted Power Law } \\
& Normal DoS & Probe R2L & U2R & $\begin{array}{l}\text { Weighted } \\
\text { Average }\end{array}$ \\
\hline $10 \%$ & 0.001 & 0.004 & 0.019 & --- & 0.03 & 0.0017 \\
\hline $20 \%$ & 0.008 & 0.001 & 0.005 & --- & 0.03 & 0.0009 \\
\hline $50 \%$ & 0.003 & 0.001 & 0.005 & --- & 0.01 & 0.0005 \\
\hline
\end{tabular}

It can be seen from the above tables that the prediction power is generally improved as more samples are used for the fitting process. For instance the prediction error of the power-law with respect to the Normal class is 0.007 when only $10 \%$ of the dataset is used for constructing the learning curve, and this error drops to 0.001 when $50 \%$ of the dataset is used for that construction.

It is also important to observe that the power law model tends to outperform the shifted power law in terms of the prediction error. This is clear in the majority classes Normal, DoS, and Probe. However this observation does not hold for minority class U2R due to the instability of their learning.

\section{CONCLUSIONS}

In this paper, we investigated the relation between the size of training set and the performance of an intrusion detection system working on NSL-KDD dataset. Our basic tool for this purpose is non-linear regression models. A model should be good in predicting performance as well as fitting performance. We compared two power law models and tested their fitting performance as well as their predicting performance. The curve fitting procedure was evaluated on each class in our intrusion dataset. It was shown that the number of instances for a given class greatly affects the fitting process. As expected, due to its flexibility, the three parameters shifted power law yields a better fit than its power law counterpart. However, it failed to fit the U2R class due to the very small number of records in this class.

We also investigated using the learning curve to predict the classifier performance when it is trained on large samples. For this purpose, we used only a small training portion of the dataset to construct a part-length learning curve by applying curve fitting using one of the two power-law models. The part-length curve is then extrapolated to a full-length curve and compared to real performance data at larger samples. This experiment was performed for random data portions of sizes $10 \%, 20 \%$, $50 \%$ of the original dataset, and using the two power-law models. In every case, the average prediction error is evaluated for each class by averaging the absolute differences between the predicted accuracies on one hand, and the observed accuracy when the classifier is really trained on the large sizes on the other hand. These experiments were conducted using JAVA language along with the WEKA machine learning tool. We find that the prediction power generally increases as more samples are used for the fitting process. Our experiments reveal also that the power law model with fewer parameters outperforms the shifted power law in terms of predicting the classifier performance at larger sample sizes.

Our future work includes considering other fitting models and more intrusion datasets. Also, we plan to study more classifiers other than the probabilistic Naive Bayes.

\section{REFERENCES}

[1] G. R. L. Figueroa, Q. Zeng-Treitler, S. Kandula, and L. H. Ngo, "Predicting sample size required for classification performance," BMC Medical Informatics and Decision Making, vol. 12, p. 8, Feb 2012.

[2] C. Perlich, Learning Curves in Machine Learning, pp. 
577-580. Boston, MA: Springer US, 2010.

[3] B. Gu, F. Hu, and H. Liu, Modelling Classification Performance for Large Data Sets, pp. 317-328. Berlin, Heidelberg: Springer Berlin Heidelberg, 2001.

[4] G. James, D. Witten, T. Hastie, and R. Tibshirani, An Introduction to Statistical Learning: With Applications in $R$, vol. 103 of Springer Texts in Statistics. Springer New York, 2013.

[5] C. Brezinski and M. Zaglia, Extrapolation Methods: Theory and Practice, vol. 2 of Studies in Computational Mathematics. Elsevier, 2013.

[6] W. Bul'ajoul, A. James, and M. Pannu, "Improving network intrusion detection system performance through quality of service configuration and parallel technology," Journal of Computer and System Sciences, vol. 81, no. 6, pp. 981-999, 2015. Special Issue on Optimisation, Security, Privacy and Trust in E-business Systems.

[7] N. Khamphakdee, N. Benjamas, and S. Saiyod, "Improving intrusion detection system based on snort rules for network probe attacks detection with association rules technique of data mining," Journal of ICT Research and Applications, vol. 8, no. 3, pp. 234-250, 2015.

[8] A. Stetsko, T. Smolka, V. Matyáš, and M. Stehlík, Improving Intrusion Detection Systems for Wireless Sensor Networks, pp. 343-360. Cham: Springer International Publishing, 2014.

[9] M. Govindarajan, "Hybrid intrusion detection using ensemble of classification methods," International Journal of Computer Network and Information Security (IJCNIS), vol. 6, no. 2, pp. 45-53, 2014.

[10] K. Atefi, S. Yahya, A. Y. Dak, and A. Atefi, A hybrid intrusion detection system based on different machine learning algorithms, pp. 312-320. Kedah, Malaysia: Universiti Utara Malaysia, 2013.

[11] Y. Wahba, E. ElSalamouny, and G. Eltaweel, "Improving the performance of multi-class intrusion detection systems using feature reduction," International Journal of Computer Science Issues (IJCSI), vol. 12, no. 3, pp. 255$262,2015$.

[12] K. Bajaj and A. Arora, "Improving the performance of multi-class intrusion detection systems using feature reduction," International Journal of Computer Science Issues (IJCSI), vol. 10, no. 4, pp. 324-329, 2013.

[13] V. Bolón-Canedo, N. Sánchez-Maroño, and A. AlonsoBetanzos, "Feature selection and classification in multiple class datasets: An application to kdd cup 99 dataset," Expert Systems with Applications, vol. 38, no. 5, pp. 5947- 5957, 2011.

[14] S. Mukherjee and N. Sharma, "Intrusion detection using naive bayes classifier with feature reduction," Procedia Technology, vol. 4, pp. 119-128, 2012. 2nd International Conference on Computer, Communication, Control and Information Technology (C3IT-2012) on February 25-26, 2012.

[15] J. Song, Z. Zhu, P. Scully, and C. Price, "Modified mutual information-based feature selection for intrusion detection systems in decision tree learning," Journal of Computer, vol. 9, no. 7, pp. 1542-1546, 2014.

[16] L.-S. Chen and J.-S. Syu, Feature Extraction based Approaches for Improving the Performance of Intrusion Detection Systems, pp. 286-291. International Association of Engineers (IAENG), 2015.

[17] S. Singh, S. Silakari, and R. Patel, An efficient feature reduction technique for intrusion detection system, pp. 147-153. IACSIT Press, Singapore, 2011.

[18] Y. Bhavsar and K. Waghmare, "Improving performance of support vector machine for intrusion detection using discretization," International Journal of Engineering Research and Technology (IJERT), vol. 2, no. 12, pp. 2990-2994, 2013.

[19] G. M. Foody, A. Mathur, C. Sanchez-Hernandez, and D. S. Boyd, "Training set size requirements for the classification of a specific class," Remote Sensing of Environment, vol. 104, no. 1, pp. 1-14, 2006.

[20] G. M. Foody and A. Mathur, "Toward intelligent training of supervised image classifications: directing training data acquisition for svm classification," Remote Sensing of Environment, vol. 93, no. 1, pp. 107-117, 2004.

[21] A. V. Carneiro, "Estimating sample size in clinical studies: Basic methodological principles," Rev Port Cardiol, vol. 22, no. 12, pp. 1513-1521, 2003.

[22] J. Cohen, Statistical Power Analysis for the Behavioural Sciences (2nd Ed.). Lawrence Erlbaum Associates, 1988.

[23] K. K. Dobbin, Y. Zhao, and R. M. Simon, "How large a training set is needed to develop a classifier for microarray data?," Clinical Cancer Research, vol. 14, no. 1, pp. 108-114, 2008.

[24] S.-Y. Kim, "Effects of sample size on robustness and prediction accuracy of a prognostic gene signature," $B M C$ Bioinformatics, vol. 10, no. 1, p. 147, 2009.

[25] V. Popovici, W. Chen, B. D. Gallas, C. Hatzis, W. Shi, F. W. Samuelson, Y. Nikolsky, M. Tsyganova, A. Ishkin, T. Nikolskaya, K. R. Hess, V. Valero, D. Booser, M. Delorenzi, G. N. Hortobagyi, L. Shi, W. F. Symmans, and L. Pusztai, "Effect of training-sample size and classification difficulty on the accuracy of genomic predictors," Breast Cancer Research, vol. 12, no. 1, p. R5, 2010.

[26] L. Kanaris, A. Kokkinis, G. Fortino, A. Liotta, and S. Stavrou, "Sample size determination algorithm for fingerprint-based indoor localization systems," Computer Networks, vol. 101, pp. 169-177, 2016. Industrial Technologies and Applications for the Internet of Things.

[27] C. Beleites, U. Neugebauer, T. Bocklitz, C. Krafft, and J. Popp, "Sample size planning for classification models," Analytica Chimica Acta, vol. 760, pp. 25-33, 2013.

[28] N. B. Amor, S. Benferhat, and Z. Elouedi, "Naive bayesian networks in intrusion detection systems," in Workshop on Probabilistic Graphical Models for Classification, 14th European Conference on Machine Learning (ECML), p. 11, 2003.

[29] I. Rish, "An empirical study of the naive bayes classifier," in IJCAI 2001 workshop on empirical methods in artificial intelligence, vol. 3, pp. 41-46, IBM New York, 2001.

[30] S. Mukherjee, P. Tamayo, S. Rogers, R. Rifkin, A. Engle, C. Campbell, T. R. Golub, and J. P. Mesirov, "Estimating dataset size requirements for classifying dna microarray data," Journal of Computational Biology, vol. 10, no. 2, pp. 119-142, 2003.

[31] C. Cortes, L. D. Jackel, S. A. Solla, V. Vapnik, and J. S. Denker, "Learning curves: Asymptotic values and rate of convergence," in Advances in Neural Information Processing Systems 6 (J. D. Cowan, G. Tesauro, and J. Alspector, eds.), pp. 327-334, Morgan-Kaufmann, 1994.

[32] G. H. John and P. Langley, "Static versus dynamic sampling for data mining," in Proceedings of the Second International Conference on Knowledge Discovery and Data Mining, KDD'96, pp. 367-370, AAAI Press, 1996.

[33] H. Motulsky and A. Christopoulos, Fitting Models to Biological Data Using Linear and Nonlinear Regression: A Practical Guide to Curve Fitting. Oxford University Press, 2004

[34] H. J. Motulsky and L. A. Ransnas, "Fitting curves to data 
using nonlinear regression: a practical and nonmathematical review," FASEB journal : official publication of the Federation of American Societies for Experimental Biology, vol. 1, no. 5, p. 365374, 1987.

[35] "Nsl-kdd data set." https://web.archive.org/web/20150205070216/http://nsl.cs .unb.ca/NSL-KDD/. [Online; accessed 21-Sep-2016].

[36] M. Tavallaee, E. Bagheri, W. Lu, and A. A. Ghorbani, "A detailed analysis of the kdd cup 99 data set," in Proceedings of the Second IEEE International Conference on Computational Intelligence for Security and Defense Applications, CISDA'09, (Piscataway, NJ, USA), pp. 5358, IEEE Press, 2009.

[37] E. Frank, M. Hall, G. Holmes, R. Kirkby, B. Pfahringer, I. $\mathrm{H}$. Witten, and L. Trigg, Weka-A Machine Learning Workbench for Data Mining, pp. 1269-1277. Boston, MA: Springer US, 2010.

[38] K.-C. Khor, C.-Y. Ting, and S. Phon-Amnuaisuk, The Effectiveness of Sampling Methods for the Imbalanced Network Intrusion Detection Data Set, pp. 613-622. Cham: Springer International Publishing, 2014.

[39] E. A. Rodríguez, "Regression and anova under heterogeneity," Master's thesis, Southern Illinois University, Carbondale, Illinois, US, 2007.

[40] L. Janson, W. Fithian, and T. J. Hastie, "Effective degrees of freedom: a flawed metaphor," Biometrika, vol. 102, no. 2, pp. 479-485, 2015.

[41] B. Efron, "How biased is the apparent error rate of a prediction rule?," Journal of the American Statistical Association, vol. 81, no. 394, pp. 461-470, 1986.

[42] R. C. Quinino, E. A. Reis, and L. F. Bessegato, "Using the coefficient of determination $\mathrm{r} 2$ to test the significance of multiple linear regression," Teaching Statistics, vol. 35, no. 2, pp. 84-88, 2013.

\section{Authors' Profiles}

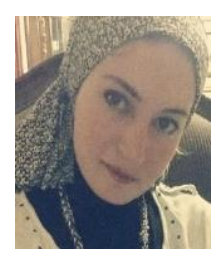

Yasmen Wahba Born in Ismailia city, April 1987, received the bachelor degree in 2008 from Suez Canal University, faculty of Computers and Informatics, Computer Science department, Ismailia, Egypt. Received the M.Sc. degree in computer science in 2016. Currently a teaching assistant and a Ph.D. student in Suez Canal University. Research expertise and interests include data mining, computer networks security, and scientific visualization.

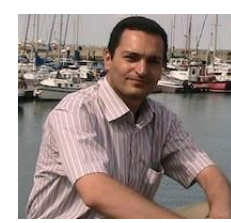

Ehab ElSalamouny Born in Damietta city, July 1976, received the bachelor degree in Computer Engineering from Cairo University, Egypt. Received the M.Sc. degree in Computer Engineering from Cairo University in 2006, and Ph.D. degree in Computer Science from Southamption University, UK, in 2011. Currently an assistant professor in Suez Canal University. Research expertise and interests include machine learning, computational trust and reputation, privacy, and security.

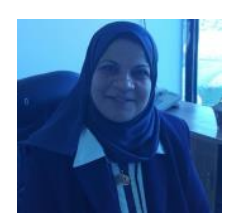

Ghada EITaweel Born in Cairo. Received the Ph.D. degree in 2005 from Cairo University. In 2011, received the Associate Professor degree. Currently a professor in Suez Canal University, Computer Science department. Research expertise and interests include image processing, data fusion and image classification.

How to cite this paper: Yasmen Wahba, Ehab ElSalamouny, Ghada ElTaweel,"Estimating the Sample Size for Training Intrusion Detection Systems", International Journal of Computer Network and Information Security(IJCNIS), Vol.9, No.12, pp.1-10, 2017.DOI: 10.5815/ijcnis.2017.12.01 